\title{
DIAGNÓSTICO PARA LA ELABORACIÓN DE UN PLAN PROSPECTIVO PARA LA PUESTA EN MARCHA DE LA CADENA PISCÍCOLA, UNA APUESTA PARA EL DESARROLLO SOCIOECONÓMICO DEL DEPARTAMENTO DE SUCRE AL 2020
}

\author{
Fabián José Mendoza Stave ${ }^{1}$
}

Recibido: 15 de mayo de 2013 Aceptado: 12 de agosto de 2013

\section{Resumen}

La baja tecnificación y mínimas producciones de la piscicultura se han convertido en debilidad en el departamento Sucre cuyas producciones de las especies Bocachico, Tilapia, Cachama son mínimas, a pesar que en su geografía pasan ríos, ciénagas, además de contar con el Golfo de Morrosquillo como fuente importante para este tipo de explotación. Ante este escenario, es necesario profundizar en el sector piscícola en Sucre y realizar el análisis de los factores que los inciden, pero también, de sus potencialidades con el ánimo de definir estrategias prospectivas para la consolidación del sector y de su articulación eficiente alrededor de la cadena.

Para la obtención de datos en la investigación se realizó un listado de expertos en el tema piscícola y desarrollo regional, se identificaron los actores, las variables más importantes del problema, las políticas y programas de apoyo a nivel nacional que inciden en el desarrollo del sector, se realizó un análisis de estudios de la situación actual del sector y se definieron los futuros escenarios y estrategias prospectivas para la puesta en marcha de la cadena. Fruto de esta investigación se presentará una síntesis del estado actual y una mirada prospectiva del sector piscícola para el departamento de Sucre.

Palabras claves: prospectiva, piscicultura, escenario, futuro, competitividad.

1 Administrador de empresas, especialista en Gerencia Pública, especialista en pedagogía para el desarrollo del aprendizaje autónomo, Magister en Recursos Humanos y organizacional, Líder de cadena de formación ECACEN Cead Corozal, Universidad Nacional Abierta y a Distancia - UNAD, fabian.mendoza@unad.edu.co, Colombia 


\title{
DIAGNOSIS FOR THE PREPARATION OF A PROSPECTIVE PLAN FOR THE IMPLEMENTATION OF THE CHAIN fish, A BET FOR ECONOMIC DEVELOPMENT DEPARTMENT TO SUCRE 2020.
}

\begin{abstract}
The low technology and low productivity of farming have become constantin some departments, as is the case of Sucre department, whose production sarequite minimal Bocachico, red tilapia and gray, Cachama despite the geographyth at they spend three majorrivers, larges wamps, natural and artificial ponds, in addition to the Gulf of Morrosquillo as important sources this type of exploitation. Given this scenario, It is necessary to deepen th estate of Sucre and fish farming sector in your state analysis of the factors that in fluencebutal so their potential with the aim to identify forward-looking strategies for the consolidation of the sector and its efficient coordination around fish chain.

To obtain data on the investigation a list of fish subject experts and regional development, were identified actors involved, the most important variables of the problem, policies and programs to support national development affecting sector, was conducted an analysis of studies of the current situation of the sector and defined the prospective future scenarios and strategies for implementation of the chain. The result of this research was presented, elements of the history of farmingin Colombia and the department, is a summary of current status and look forward, the fish sector for the department of Sucre
\end{abstract}

Keywords: prospective, aquaculture, stage, future competitiveness..

\section{Introducción}

La aplicación del conocimiento y desarrollo tecnológico se han convertido en factor de producción, desarrollo social y económico en especial en los países en vía de desarrollo. En Colombia, el esfuerzo tecnológico por parte del Ministerio de Agricultura y Desarrollo Rural por volver más competitivo el sector agropecuario se ha basado en la definición y puesta en marcha de conglomerados que según sus características particulares han recibido el nombre de sectores, cadenas y clúster. Permitiendo además, la inclusión de diferentes actores o eslabones desde la producción hasta el consumidor final.

En el departamento de Sucre, los avances tecnológicos del sector son notables en el subsector ganadero. Pero el subsector piscícola los avances tecnológicos son insignificantes a pesar que el departamento de Sucre cuenta con muchas ventajas en sus aguas continentales, marítimas y su ubicación geográfica para el crecimiento y desarrollo del sector. En este sentido el desarrollo del «Diagnostico para el diseño de un Plan prospectivo para la puesta en marcha de la cadena piscícola, una apuesta para el desarrollo socioeconómico del departamento de Sucre al 2020", se constituye, en una propuesta esencial para articular e integrar a los distintos actores e instituciones regionales y nacionales en la búsqueda de una visión de futuro competitiva de este subsector agropecuario, a partir del análisis de sus debilidades, de las oportunidades de su entorno, identificación de sus actores y variables claves, definición de escenarios y en especial de la construcción de estrategias que puedan incluirse en los planes, 
programas y proyectos de las instituciones en Sucre en el corto, mediano y largo plazo. En las últimas décadas se ha evidenciado el auge de la acuicultura y piscicultura a nivel mundial, el mejoramiento de los procesos tecnológicos y la visión empresarial de los centros productores piscícolas en algunos países ha permitido que algunos continentes y regiones hayan escalado ventajosamente a superficie de mercados internacionales con relación a otros.

En Colombia, en el año 2006 se produjeron 48.532 toneladas, de los cuales 23.146 son de Tilapia Roja y Nilótica, siendo los departamentos de mayor producción Huila, Valle, Risaralda, Llanos Orientales y Antioquia. En sucre según el Plan de Desarrollo Departamental de Sucre «Liderazgo Social y Confianza» 2008 - 2011, en el diagnóstico realizado a los sectores agropecuarios, pesqueros, acuícola, forestal y minero del departamento de sucre, en el periodo 2000 - 2005 este sector decreció su participación en el PIB nacional pasando de $0,81 \%$ a $0,77 \%$ y a nivel departamental su participación en el PIB en el periodo 2005 - 2006 decreció pasando de $23,20 \%$ a $21.67 \%$. Siendo la actividad que más contribuyó con el PIB en este año 2006, la venta de animales vivos y productos animales con un $59,29 \%$, productos agrícolas $36.63 \%$, productos de la pesca $2.42 \%$ y silvicultura $1.66 \% 1$.

En el año 2007 - 2008 el sector piscícola mantuvo constante sus producciones y se identificó como factores de este decrecimiento a los mínimos componentes tecnológicos y comerciales como son: manejo tradicional, bajos rendimientos y productividad, falta de un centros de acopio - plantas de eviscerado y transformación especializado que permita que las producciones en el departamento se realice un manejo de excelente calidad del producto bajo la cadena de frío y a los productores pequeños y medianos articular sus producciones a la cadena piscícola insertándose hacia mercados de superficies regionales y nacionales. Según infor- mación suministrada por la Unidad Regional de Planeación Agropecuaria URPA año 2007, en el departamento de sucre la baja producción y rendimiento del sector se refleja en las siguientes cifras: número de peces sembrados en jaulas y estanques en total de 958,320 peces y se cosecho un total de 501.361 peces (\% perdida de la actividad 47,6), produciendo 284 toneladas de carne de pescado en su totalidad, distribuidas así: Bocachico 117 toneladas, Cachama estanque 93 toneladas, Tilapia Nilótica jaula 16 toneladas y estanque 14 toneladas, Tilapia Roja jaula 13 toneladas y estanque 21 toneladas y Sábalo estanque 10 toneladas ${ }^{2}$. Es de anotar, que si bien existe unas políticas claras desde el gobierno nacional para el apoyo y fomento a la cadena piscícola a nivel nacional, y desde la gobernación de Sucre, a través del plan departamental de desarrollo 2008 - 2011 «Liderazgo Social y Confianza» se han planteado estrategias de apoyo al sector agropecuario y pesquero entre las que se encuentra, a) Implementar y legalizar la cadena productiva con el apoyo del sector público y privado y las alianzas productivas, b) Mejorar la competitividad de la producción, formalizar alianzas institucionales y con actores sociales con perfil empresarial y abrir nuevos mercados y c) Impulsar la generación de ingreso y la seguridad alimentaria en el departamento. El no contar con un estudio actualizado sobre el estado del sector piscícola y de las ventajas/desventajas que este cuenta en cada una de las 5 subregiones del departamento, no ha permitido la construcción de acciones o proyectos claves que se inserten dentro de los planes y programas departamentales que hagan uso del potencial hídrico, que tecnifique los diferentes eslabones de la cadena productiva piscícola para su fortalecimiento y competitividad a nivel regional y nacional ${ }^{3}$.

Como consecuencia de lo antes mencionado, he encontrado que el departamento de Sucre

\footnotetext{
2 Documento de coyuntura de la Unidad de Planeación Agropecuaria de la gobernación de Sucre URPA 2008.
}

Plan de desarrollo departamental de Sucre 2008 - 2011, "Liderazgo Social y Confianza". 
no ha podido realizar un verdadero análisis del sector, y mucho menos la definición de verdaderas estrategias prospectivas para el sector hacia su tecnificación, crecimiento y competitividad. Conllevando por el contrario que las acciones y proyectos a incluir al interior de los planes y programas departamentales respondan al aprovechamiento de recursos nacionales e internacionales, con intervenciones aisladas sin que exista una concreción de una visión holística para el sector de los distintos actores sociales, que conlleve a esta actividad a consolidarse como un sector empresarial, económico y social para el departamento y la región Caribe. Por ello contamos con un objetivo general que es: Diseñar un plan prospectivo para la puesta en marcha y competitividad de la cadena piscícola en el departamento de Sucre al 2020. Así mismo, unos objetivos específicos, entre otros, Realizar un diagnóstico real sobre el estado del sector piscícola en el departamento de Sucre. Definir las variables que permitan realizar un análisis actual y prospectivo del sector piscícola del departamento. Identificar los actores que pueden incidir directamente en la implementación de las estrategias de cambio positivo al sector en la actualidad y en el futuro. Construir escenarios que permitan la competitividad del sector y el fortalecimiento socioeconómico del departamento en el futuro.

\section{DESARROLLO}

La competitividad empresarial en los mercados nacionales e internacionales ante el fenómeno de la globalización es cada vez más exigente, hoy por hoy las empresas que una vez fueron únicas y líderes se han visto en la necesidad de replantear sus estrategias productivas, innovación tecnológica, de marketing y alianzas para mantenerse y ser competitivas en los mercados regionales, nacionales e internacionales. Este fenómeno está conllevando que los diferentes países y sus departamentos o estados sin importar su nivel de desarrollo se vean en la necesidad de estudiar las nuevas tendencias de los mercados y a replantear sus planes de desarrollo basados en una visión prospectivas del sector y de la demanda, considerando como parte fundamental de su estudio las oportunidades del medio (recursos naturales, medios de producción, ubicación geográfica, capacidades productiva y tecnológica y capital humano).

En este orden de ideas, el departamento de Sucre cuya economía se centra en el agro debido a la riqueza y diversidad de sus ríos, cuerpos de aguas, mar y paisaje, no escapa a esta realidad, y si bien es cierto que frente al sector piscícola se vienen realizando algunas acciones muy aisladas a través de algunos proyectos, estos, en la mayoría de los casos han respondido a iniciativas productivas de seguridad alimentaria o pedagógicas siendo muy pocas las iniciativas de producción empresarial que involucre en sus procesos el mercadeo y comercialización y mucho menos la transformación de los diferentes productos y la articulación los diferentes componentes que realmente constituyen una cadena piscícola.

Por lo anterior, la realización del Diagnóstico para la elaboración de un Plan prospectivo para la puesta en marcha de la cadena piscícola, una apuesta para el desarrollo socioeconómico del departamento de sucre al 2020 se justifica ya que permite conocer el estado del sector piscícolas de Sucre, las principales variables y actores claves para la puesta en marcha del sector y en especial permitirá la construcción del escenario ideal y de estrategias para alcanzarlo.

En la actualidad existen unas 868 unidades productivas, de las cuales 837 , es decir el $95 \%$ realizan sus producciones en estanques y solo un $4 \%$ alcanza a producir sus peces en jaulas, siendo las especies producidas en las jaulas la tilapia roja, especialmente, lo que indica lo incipiente de la practica en el departamento sucreño ${ }^{4}$.

\footnotetext{
${ }^{4}$ Documentos de Coyuntura de la Unidad Regional de Planeación Agropecuaria, de la
} Gobernación de Sucre URPA 1997 a 2008. 
El Diagnóstico Piscícola del Departamento de Sucre realizado dentro del proyecto denominado «Inventario Acuícola Nacional» de la subgerencia Pecuaria del Instituto Colombiano Agropecuario, ICA, se realizó en el periodo comprendido entre enero de 2005 a diciembre de 2006, tiempo en el cual se visitaron todos los predios (359 predios), sin importar el nivel de tecnificación, infraestructura existente, manejo de la explotación ni la finalidad de la producción, esta investigación se realizó en todos los municipios menos el Roble por problemas de orden público, y fue realizada mediante el Convenio CN - 067 - 2004 entre la Universidad y el ICA - Sucre.

\section{a. Asistencia Técnica y Recurso Humano} para el sector Piscícola en sucre, según el diagnóstico al sector Piscícola en Sucre, UniSucre - ICA, 2006. Se encontró que de los 359 predios piscícolas encuestados el $17.3 \%$ de los predios reciben asesoría técnico profesional; realizada en gran parte por los técnicos de las UMATAS. El resultado de estas asesorías es, en muchos casos cuestionable, pues los técnicos asisten a los productores, en su mayoría, cuando se realiza la siembra de los alevinos, que muchas veces ellos mismos suministran, y nunca más vuelven al sitio del cultivo, es por esto que al final del proceso, al momento de cosechar, generando muchas pérdidas. Sin embargo algunos productores buscan asesorías con personas particulares capacitadas para alcanzar resultados son más favorables.

b. Procedencia de la Semilla o Alevinos, En el diagnóstico realizado existe en el departamento de Sucre dos fincas donde se comercializan a partir de la compra las larvas y post larvas en el departamento de Córdoba (en las ciudades de Montería y Lorica), en algunos casos al Centro de investigaciones piscícolas de la Universidad de Córdoba (CINPIC); una vez en las fincas realizan todos los procesos hasta sacar al mercado alevinos de muy buena cali- dad. Estas fincas son la Piscícola Maraca en el municipio de Corozal, y la asociación ASOMUPROSAN ubicada en la vereda San Jaime en el municipio de Los Palmitos..

c. Tipos de Cultivos en Sucre, De igual forma el diagnóstico al sector piscícola realizado por ICA - UniSucre, 2006 al sector piscícola, logro identificar que en sucre el sistema intensivo abarca el $0,28 \%$ de los predios piscícolas encuestados, esto es solo una (1) granja de las 359 granjas encuestadas; esta es una granja de Alevinaje que comercializo en el año 20064 millones de alevinos (Bocachico y Cachama Negra) ubicada en el municipio de Corozal; la alimentación es a base de alimento balanceado, además se realiza fertilización orgánica e inorgánica.

El sistema Semi-Intensivo abarca el $18.66 \%$ de los predios piscícolas; son predios en los que se realiza una alimentación con alimento concentrado hasta un periodo de 2 - 3 meses y complementan con una alimentación con abonos orgánicos y otros productos naturales (guayaba, totumo, maíz cocido y yuca seca). De este $18.66 \%$, existe un $22.8 \%$ que además del alimento balanceado comercial, suministran a los cultivos estiércol bovino, equino y porcino, el $2.8 \%$ suministran balanceado comercial, estiércol y otros productos naturales, el $1.4 \%$ suministran balanceado comercial frutas y otros productos ya mencionados, el $5.7 \%$ suministran una alimentación a cultivo de sábalos a base de peces vivos (es importante mencionar que son predios en el municipio de San Onofre en los cuales se está realizando la practica con Sábalo a nivel experimental).

d. Tipo de Instalaciones en Sucre, de los 359 predios piscícolas que participaron de diagnóstico realizado por el ICA y UNISUCRE, en el 2006, se encontró que existían 378 estanques con un área total de $899.096 \mathrm{~m}^{2}$ y 384 represas con una área de $1.524 .208 \mathrm{~m}^{2}, 36$ jagüeyes con 
$27.200 \mathrm{~m}^{2}$ y un total de 10 Jaulas con un volumen de $40.4 \mathrm{~m}^{3}$; la mayoría de las instalaciones son represas con mayor presencia en los municipios de Majagual, Morroa y Sucre seguidos por estanques con presencia en los municipios de Coveñas, San Marcos y Corozal, jagüeyes con mayor presencia solo en los municipios de San Onofre, Morroa y Guaranda y las jaulas las cuales se encontraron en Toluviejo y Sincelejo.

De los 378 estanques encuestados, el $7.4 \%$ no están en producción, esto significa que el área en producción es solo $868.568 \mathrm{~m}^{2}$; de las 384 represas el $29.7 \%$ no están en producción, o sea solo 1.039.915 $\mathrm{m}^{2}$ están en producción, y de los 36 jagüeyes el $44.4 \%$ no están en producción, es decir, solo $9.687 \mathrm{~m}^{2}$ están en producción. Todas las jaulas encontradas se encontraban en producción. Las razones principales del no uso de todas las instalaciones, fue el mal estado de muchas de ellas, la filtración y el desborde del agua, inundaciones, inseguridad (robo de los peces), escasez de agua por sequía, falta de semilla, entre otras. Según los informes de coyuntura de los años 2001 a 2005 de la Secretaría de Desarrollo Económico y Medio Ambiente, el departamento de Sucre pasó de 469 estanques $\left(1.241 .558 \mathrm{~m}^{2}\right)$ en el año 2001 a 1.409 (2.755.558 $\left.\mathrm{m}^{2}\right)$ en el 2005; de los cuales estaban en producción 866, es decir, el área de producción fue de $1.694 .580 \mathrm{~m}^{2}$.

\section{e. Aspectos Sanitarios y Ambientales de} la Piscicultura en Sucre, de igual forma el diagnóstico logro identificar que de los 359 predios, solo el $31.2 \%$ cumplen por lo menos con una de las siguientes normas de seguridad sanitaria y ambiental:

- Tratamiento de las aguas residuales.

- Programa de prevención sanitario.

- Programa de limpieza y desinfección de las diferentes zonas del cultivo.
- Manejo de residuos.

- Manejo, desinfección y transporte del producto.

Este $31.2 \%$ está compuesto de la siguiente forma, el $1.8 \%$ cumple con todas las normas menos con el tratamiento de las aguas residuales, el $3.57 \%$ cumple con el programa de limpieza y desinfección, manejo de residuos, y manejo, desinfección y transporte del producto, el 7.1\% cumple con el programa de limpieza y desinfección y con el manejo de residuos, el $4.46 \%$ cumple con el programa de limpieza y desinfección y con el manejo, desinfección y transporte del producto, el $2.67 \%$ cumple con el manejo de residuos y con el manejo, desinfección y transporte del producto, el $8 \%$ cumple solo con el programa de limpieza y desinfección de las diferentes zonas del cultivo, el $66.7 \%$ cumple solo con el manejo de residuos, y el $6.25 \%$ cumple solo con el manejo, desinfección y transporte del producto.

f. Producción Piscícola Encontrada, el diagnóstico realizado por ICA - UniSucre, 2006 al sector piscícola, anota que si bien se visitaron predios representativos del departamento de Sucre, los datos suministrados no pueden considerarse como datos de producción absolutos en el departamento, y que la producción registrada corresponde al periodo comprendido entre Enero de 2005 y Diciembre de 2006, como se describen en la siguiente tabla.

Consciente que el presente nos debe situar en el hoy, para la contextualización del presente del sector piscícola en el Departamento de Sucre, se ha tomado los resultados de este diagnóstico el cual es el único documento existente en el Departamento de Sucre que nos acerca a un contexto real sobre el estado de la piscicultura en el departamento de Sucre: 


\section{Metodología, teorías y resultados}

La presente investigación como tiene un eje temático prospectivo, se basa en teorías y unos métodos los cuales se nombran a continuación y dependiendo la conceptualización de cada uno se aplicaron entrevistas, encuestas y la observación directa e inmediatamente se dio aplicación a cada uno de ellos, teniendo de esta manera los resultados:
EL MÉTODO DELPHI. Consiste en la selección de un grupo de expertos a los que se les pregunta su opinión sobre cuestiones referidas a acontecimientos del futuro. Las estimaciones de los expertos se realizan en sucesivas rondas, anónimas, al objeto de tratar de conseguir consenso, pero con la máxima autonomía por parte de los participantes; es decir, poner de manifiesto convergencias de opiniones y deducir eventuales consensos.

Descripción del Cuadro de Expertos

\section{ITEM NOMBRE LARGO \\ DESCRIPCIÓN}

\begin{tabular}{|c|c|c|}
\hline $\mathbf{E}_{1}$ & AMAURY HERNÁNDEZ MERCADO & $\begin{array}{l}\text { Economista. Profesional Universitario -Asesor Proyectos Espe- } \\
\text { ciales - Gobernación de Sucre }\end{array}$ \\
\hline$E_{2}$ & ROBERTO CARLOS PINEDA & $\begin{array}{l}\text { Biólogo, Profesional Universitario de Sucre. Investi- } \\
\text { gador agenda productivay desarrollo de sucre. }\end{array}$ \\
\hline $\mathbf{E}_{3}$ & JOSÉ VICENTE BANQUET PERNA & $\begin{array}{l}\text { Ingeniero Agrónomo, coordinador proyectos piscícola-Gober- } \\
\text { nación de Sucre }\end{array}$ \\
\hline $\mathbf{E}_{4}$ & MARLON DEL VALLE CASTILLO & $\begin{array}{l}\text { Economista. Asesor Empresarial, Cámara de Comercio de } \\
\text { Sucre. }\end{array}$ \\
\hline $\mathbf{E}_{5}$ & GERARDO MIRANDA GALINDO & $\begin{array}{l}\text { Ingeniero Agrícola. Profesional universitario - funcionario ICA } \\
\text { - Sucre. }\end{array}$ \\
\hline $\mathbf{E}_{6}$ & YOLANDA PARRA & $\begin{array}{l}\text { Ingeniera piscícola. Asesora profesional proyectos piscícola } \\
\text { Montes de María - III LP }\end{array}$ \\
\hline $\mathbf{E}_{7}$ & JUAN SALCEDO ESTRADA & $\begin{array}{l}\text { Tecnólogo en producción Agropecuario. Unisucre. } \\
\text { Coordinador de proyectos piscícolas en Montes de } \\
\text { María. LP III. }\end{array}$ \\
\hline$E_{8}$ & MÁXIMO CALDERÓN CÁLIZ & $\begin{array}{l}\text { Profesional. Director Ejecutivo y Representante le- } \\
\text { gal Corporación Incubadora de Empresas de Sucre } \\
\text { - Incubar Sucre. }\end{array}$ \\
\hline$E_{9}$ & FRANCISCO BENÍTEZ RAMOS & $\begin{array}{l}\text { Representante Fundación FIDES, Proyectos pro- } \\
\text { ductivos agropecuarios y sociales para Sucre. }\end{array}$ \\
\hline$E_{10}$ & LUCELLYS RAMOS CONTRERAS & $\begin{array}{l}\text { Administradora de empresas, Universidad de Su- } \\
\text { cre. Proyectos de desarrollo social FIDES. }\end{array}$ \\
\hline $\mathbf{E}_{11}$ & JAIME BUELVAS RODRÍGUEZ & $\begin{array}{l}\text { Ingeniero pesquero, Profesional asesorárea pes- } \\
\text { quera INCODER, Sucre. }\end{array}$ \\
\hline$E_{12}$ & JOSÉ LUIS GUTIÉRREZ & $\begin{array}{l}\text { Tecnólogo en Producción agropecuaria, piscicultor, } \\
\text { Granja Integral la Fortuna- Tolú. }\end{array}$ \\
\hline$E_{13}$ & TOMAS PÉREZ MEDRANO & $\begin{array}{l}\text { Profesional en Acuicultura instructor SENA - pisci- } \\
\text { cultor y comercializador piscícola }\end{array}$ \\
\hline $\mathbf{E}_{14}$ & LINA MARÍA WADNIPAR CANO & Profesional en Acuicultura, universidad de sucre. \\
\hline$E_{15}$ & GUSTAVO HERRERA REVOLLO & Profesional en Acuicultura - ICA seccional Sucre. \\
\hline
\end{tabular}

Fuente: Elaboración propia 


\section{Encuesta a expertos}

En total se encuestaron 15 expertos acuicultores (as) y de diferentes entidades, la encuesta se fundamentó en desarrollar interrogantes a través de la misma, la cual tenía que ser respondida de manera individual por los asesores, luego los asesores la devolvía al investigador y se programó una próxima reunión. La información resultante de la encuesta se tabuló y se hizo un procesamiento para su análisis y posterior presentación de resultados en la investigación

\section{Matriz dofa - diagnóstico estratégico}

es una herramienta de análisis estratégico, que permite analizar elementos internos o externos de programas y proyectos. En síntesis: a) las fortalezas deben utilizarse, b) las oportunidades deben aprovecharse, c) las debilidades deben eliminarse y d) las amenazas deben sortearse.

\section{Debilidades del Sector Piscícola en el Departamento}

No existe un diagnostico actualizado real del estado del sector piscícola (unidades, áreas, procesos, manejo técnico, calidad del producto, capacidad, mercado otros) en el departamento; No se cuenta al interior de las instituciones con capital humano con la experiencia que oriente este sector productivo hacia la competitividad; No existe una visión empresarial y regional sobre cada uno de los componentes de la actividad piscícola e instituciones y productores; Desconocimiento técnico y falta de agremiación y articulación de los pequeños productores según particularidades de las subregiones; Falta de infraestructura para la articulación de los procesos productivos, de transformación y comercialización del sector piscícola; No se cuenta con una cadena productiva en este sector; Falta de articulación y encuentro entre los piscicultores y universidades, ICA y sector privado; Vías en mal estado y falta de transporte y equipos de transporte adecuados para su comercio a los centros de mercados.
Oportunidades del Sector Piscícola en el Departamento

Demanda insatisfecha de los mercados departamental, nacional e internacional; Políticas definidas y recursos de apoyo disponibles para el sector desde el gobierno central y desde la cooperación internacional; se cuenta con expertos en el tema de construcción de cadenas productivas piscícolas en departamentos como Huila, Meta, Valle del Cauca otros, que puede asesorar el proceso de cadena en Sucre; EI TLC y la definición de alianzas comerciales con otros países.

\section{Fortalezas del sector Piscícola en el Departamento}

Diversidad y disponibilidad del recurso hidrobiológicos continental y marítimo. Por ser una población rodeada de ríos, ciénagas y mar, existe una fuerte tradición hacia la pesca, que se puede incidir hacia la piscicultura tecnificada; Ubicación estratégica por el mar, ríos, aire hacia mercados nacionales e internacional; Abundante y diversa oferta natural en los ecosistemas; Disponibilidad de las universidades para apoyar los procesos de investigación y educación para el sector.

Amenazas del sector Piscícola en el Departamento

Falta de una planeación estratégica para la competitividad del sector, desde el gobierno central y departamental y continuidad del mismo; Contaminación de los espejos de agua continentales por factores varios; falta de garantías de entidades crediticias para el fomento y fortalecimiento del sector piscícola; Conflicto armado y actores al margen de la ley; el TLC, también puede ser vista como una amenaza si consideramos que se puede importar productos piscícola a bajos precios, lo que limitaría el crecimiento y puesta en marcha de la cadena en el departamento. 
Teniendo en cuenta el gran número de debilidades y amenazas frente a las pocas fortalezas en el sector, se hace necesario que las entidades y comunidades involucradas, conjuntamente definan estrategias que le permitan potenciar oportunidades para el fortalecimiento del sector del departamento a corto y mediano plazo.

\section{Método mic mac - análisis estructural:}

Mediante la aplicación del método MIC MAC es posible identificar las variables claves endógenas y exógenas para el futuro. Este análisis se realiza a través del grupo de trabajo compuesto por los actores y expertos con experiencia demostrada, sin que se excluya concejeros externos. Este método puede ser empleado en la reflexión para la selección de estrategias y como insumo para métodos más generales como la técnica de escenarios. En tal sentido, contribuye a la toma de decisiones porque permite identificar las variables sobre las cuales es necesario actuar para lograr los objetivos (Mera, 2005; Restrepo, 2003; Godet, 2000). A través del método MIC-MAC, se logró la identificación de las variables más motrices y más dependientes el cual se identificó a partir de la calificación de las variables por parte del grupo de expertos, para lo cual se definieron los siguientes valores 1(Débil), 2 (Mediana), 3 (Fuerte) y 4 (Potencial) de manera directa. Al analizar las se logran identificar las variables que ejercen la mayor acción directa; las cuales se identificaron con la calificación máxima. Estas calificaciones realizadas por los expertos nos permitieron establecer el nivel de jerarquía expresado en las variables con calificación potencial, fuerte, mediana y débil. En conclusión identificamos que las variables de políticas institucionales, inversión al sector, alianzas y articulaciones, recurso humano dedicado a la investigación y el desarrollo tecnológico y la apoyo interinstitucional y centros de investigación, al igual que el nivel organizativo para el sector son las variantes potenciales que representaron mayor importancia mientras que el fomento a la piscicultura y consumo per cápita y la cultura empresarial son menos fuertes, seguidas de las demás variables. Opuesto a lo anterior, se encuentran las variables de menor jerarquía, sin dejar de ser importantes como son las tecnologías de información y comunicación, la innovación en productos las cuales se encontraban con una calificación bastante baja dejándolas por fuera del nivel de influencia, por lo que pierden importancia al enfrentarse a las relaciones indirectas, esta disminución de la influencia no las clasificas como variables claves (Godet, 2000). A partir de lo anterior, identificamos como Variables de mayor jerarquía e importancia para el sector las siguientes:

\section{- Políticas institucionales tecnológicas} y de fomento al sector piscícola (PITF). Esta variable se considera influye de forma directa sobre la dinámica sectorial, ya que aglutina a su vez las variables de tipo tecnológico como son la inversión sobre en infraestructura tecnológica, recurso humano con experiencia y sobre el desarrollo tecnológico a partir de las políticas nacionales y por ende de los gobiernos locales. De esta relación se deduce que al aumentar el desarrollo tecnológico apoyado por políticas claras y planificadas, se fomentara el desarrollo del sector y por ende su competitividad y productividad en el departamento y la región Caribe.

- Recurso humano dedicado en la investigación y el desarrollo tecnológico (RHIDT): Esta variable que es incidida directamente por la variable de inversión al sector y desarrollo tecnológico, es una de las de mayor demanda y relevancia para el sector piscícola, dado a que a pesar de las oportunidades potenciales existentes en nuestro departamento por su biodiversidad y su hidrografía, no se cuenta con el recurso humano preparado y dedicado a poner en marcha esta iniciativa. 
- Nivel organizativo y de agremiación del sector piscícola (NOA): Este item es influido por la mayoría de las variables y recibe mayor influencia de la variable política institucional tecnológica y de fomento, de la variable de orden público, y en menor proporción de las variables comunicación e innovación de productos, aun cuando se cree que la variable cultura empresarial de los piscicultores y pescadores. esta variable tiene una fuerte influencia fuerte sobre el desarrollo tecnológico y dinámica del sector. Ya que al fortalecerse las organizaciones y agremiaciones de manera integral se deduce que puede existir la definición de alianzas y articulación entre los distinto eslabones de la cadena piscícola y la conformación de clúster entre los actores de manera focaliza$\mathrm{da}$, considerando las potencialidades de las diferentes subregiones, lo que conllevara a un mejoramiento de la productividad y competitividad del sector.

- Centros de investigación y desarrollo tecnológico (CIDT): variable de mucho valor para el sector, vista desde muchos puntos de vista (investigación, recuperación y conservación de especies, mejoramiento genético, centro de enseñanza entre otros) ya que incide altamente sobre las variables de capacitación del recurso humano, cultura empresarial, recurso humano dedicado a la investigación y innovación de productos y tecnología para los procesos al interior de la cadena productiva piscícola.

- Nivel de Desarrollo tecnológico (NDT): Es a partir de la implementación de estas es que el sector puede avanzar hacia una proyección y desarrollo en la búsqueda de la consolidación de verdadera cadena productiva en el departamento.

- Apoyo interinstitucional (Al): No es posible avanzar hacia la solución de problemas o ne- cesidades, sin la participación de los actores e instituciones privadas y territoriales.

\section{- Alianzas y articulación entre los actores} del sector (AAAS): Esta variable debe ser entendida como el espacio de interacción, articulación y de toma de decisiones entre todos los actores que intervienen en los distintos eslabones de la cadena productiva como son: Los productores de alevinos, cebadores, plantas de procesamiento o transformación, pequeños y grandes comerciantes y consumidores finales y empresas exportadoras comerciales y de pequeñas plantas de alimento concentrados, para articularse y conformar clúster o cadenas en aras a considerar un comercio justo y solidario que permita fortalecer tecnológicamente el sector.

\section{Método mactor - juego de actores.}

Este método de Prospectiva complementa el trabajo de identificación de variables claves para el futuro, es decir, definir los Actores que jugarán el papel principal en la construcción de dicho futuro.

El método MACTOR se enfoca fundamentalmente en la determinación de las motivaciones, conflictos y posibles alianzas estratégicas entre los Actores de cara al futuro ${ }^{1}$. Luego se pasa a un Análisis del juego de actores, llamado «Análisis de relación entre actores», el cual busca valorar las relaciones de fuerza entre los actores y estudiar sus convergencias y divergencias con respecto a un cierto número de posturas y de objetos asociados. Se identificaron 11 Actores en total y su correspondiente posicionamiento en relación con los 11 objetivos estratégicos lo cual permite analizar las oportunidades de concertación entre los actores y prever los conflictos potenciales que deben ser canalizados para construir alianzas indispensables para la construcción de los escenarios.

${ }^{5}$ Prospectiva estratégica, el futuro escenario, Carlos William Mera Rodríguez 2005 Caj de Herramientas de la prospectiva Estratégica, Michel Godet 2000. 


\section{ACTORES DE MAYORES INFLUENCIAS PARA EL SECTOR PISCÍCOLA.}

\begin{tabular}{|cll} 
N $^{\circ}$ & \multicolumn{1}{c}{ Instituciones y Empresas Públicas y Privadas } & \multicolumn{1}{c}{ N C } \\
\hline 1 & Gobernación de Sucre y Alcaldías Municipales. & GSM \\
\hline 2 & Universidades públicas y privadas. & UPP \\
\hline 3 & ONG de cooperación nacional e internacional. & ONG,S \\
4 & Servicio Nacional de Aprendizaje SENA. & SENA \\
\hline 5 & $\begin{array}{l}\text { Instituto Colombiano para el Desarrollo de la Ciencia } \\
\text { y la Tecnología, COLCIENCIAS. }\end{array}$ & COLC \\
\hline 6 & Instituto Colombiano de Desarrollo Rural INCODER & INCOD \\
7 & Instituto Colombiano Agropecuario ICA & ICA \\
\hline 8 & $\begin{array}{l}\text { Corporación para el Desarrollo Sostenible de la Mojana } \\
\text { y el San Jorge CORPOMOJANA. }\end{array}$ & CMOJ \\
\hline 9 & Cámara de Comercio & CC \\
\hline 10 & Empresas y asociaciones productoras Piscícolas & AEPP \\
11 & Empresas comercializadoras de pescados a nivel departamental & COMP \\
\hline 12 & $\begin{array}{l}\text { Asociación de productora de concentrados para Animales } \\
\text { CONCEPAL - Palmira - Toluviejo. }\end{array}$ & CONC \\
\hline
\end{tabular}

Fuente: Elaboración propia.

Método de construcción de escenarios.

El método de escenarios es una técnica que tiene por objeto definir un estado futuro de un sistema conocido actualmente (por lo menos parcialmente) e indicar los distintos procesos que permiten pasar del estado presente a la imagen futura. Los escenarios son en definitiva un instrumento de simulación que permite mejorar nuestra comprensión de las consecuencias a largo plazo, de las tendencias de políticas existentes o potenciales, y sus interacciones. El futuro lo clasificamos en tres escenarios posibles, el escenario más probable y dos escenarios alternativos.

Un escenario es hipotético (describe algún futuro posible), es selectivo (representa algunos aspectos de la realidad), es limitado (contiene una número determinado de variables) y está conectado (interconexión entre situaciones). Los escenarios también pueden valorarse en términos de probabilidad. Si bien no hay un modelo único de elaboración de escenarios, se puede aceptar como válido el proceso siguiente. En el método de escenarios se distinguen dos fases principales: 1 . La construcción de la base y 2 . La elaboración de escenarios propiamente dicha (Caballano, s.f.; Mera, 2005; Godet, 2000; Franco, 2003)..

\section{Agrupación de variables:}

Para diseñar los escenarios se toma como fundamentos la teoría de Peter Schwartz. Según la teoría, inicialmente se debe realizar una agrupación de las variables anteriores en dos categorías, considerando el nivel de calificación o jerarquía tal como aparece en el cuadro siguiente: 


\section{Unificación de Variables}

SOCIOCULTURAL - TECNOLÓGICA

POLÍTICO - ADMINISTRATIVO
1. Nivel de desarrollo tecnológico

2. Nivel organizativo y de agremiación

3. Alianzas y articulación entre actores del sector piscícola

4. Recurso humano dedicado a la investigación y al desarrollo tecnológico
1. Política institucional tecnológica y de fomento

2. Inversión al sector piscícola.

3. Apoyo interinstitucional.

4. Centros de investigación y desarrollo tecnológico

Fuente: Elaboración propia

A partir de esta agrupación y teniendo en cuenta el comportamiento de estos factores en el pasado, se puede especular que las variables analizadas, en el futuro podrán estar en una situación mejor o peor, por lo que se le atribuirá un criterio de mínimo (-) o máximo (+), dependiendo de cada situación según el nivel de influencia tendencial que cada una de estas variables tenga sobre el escenario.

\section{Elaboración del eje de schwartz}

De esta clasificación de situaciones posibles, se generan cuatro escenarios los cuales se determinan a partir de señalar el mejoramiento de la situación con el signo + o por el contrario, se señalara el empeoramiento de la situación con el signo -. Solo existe cuatro combinaciones posibles de los signos + y -, por lo tanto, dentro de los próximos 8 años (2020), las variables escogidas se sitúan en uno de los cuatro cuadrantes, según las situaciones presentes: (+ -) (-+) (--) $(++)$ que gráficamente se puede visualizar así:

Escenario Alterno 1: Ocurre la primera variable o categoría positivamente (+) Pero ocurre la segunda variable o categoría negativamente (-). Escenario Alterno 2: Ocurre la primera variable o categoría negativamente (-) Pero ocurre la segunda variable o categoría positivamente (+). Escenario Catastrófico: Ocurre la primera variable o categoría negativamente (-) ytambién ocurre la segunda variable o categoría negativamente (-). Escenario Apuesta: Ocurre la primera variable o categoría positivamente (+)'y también ocurre la segunda variable o categoría positivamente $(+)$.

Para la descripción y análisis de los escenarios, se toman como referencia para los dos ejes los dos grupos de variables anteriores que conformaron las dos grandes categorías, y se ubican en el eje así Las sociales y culturales en la segunda categoría (Godet, 2000; Franco, 2003).

Construcción de escenarios para el sector Piscícola

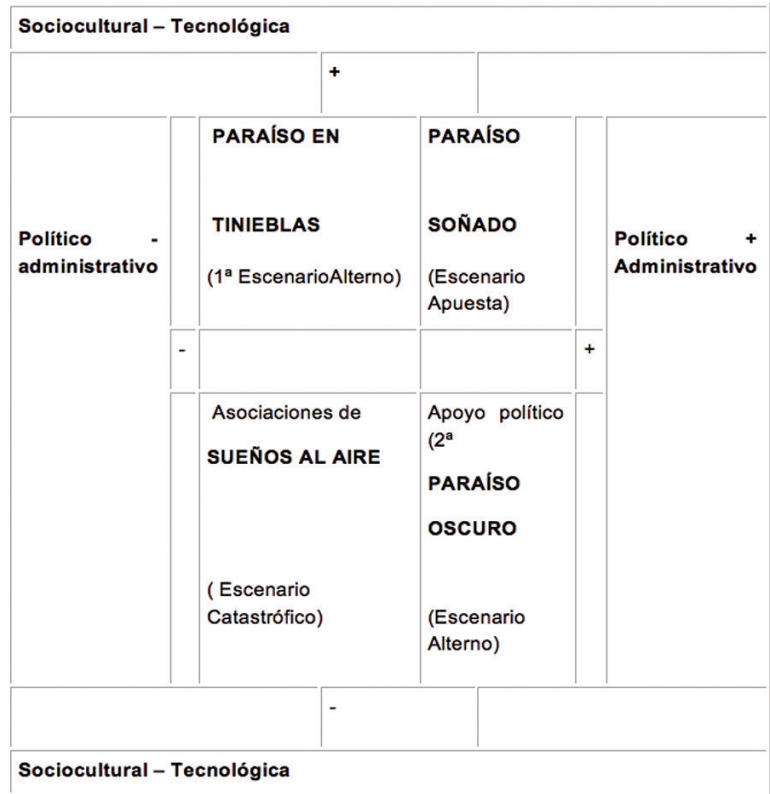

Fuente: Godet, 2003 
Paraíso Soñado - Escenario Apuesta: Agremiación del sector piscícola de Sucre sólida y compitiendo en mercados nacionales e internacionales.

Considerado como el panorama más positivo o escenario apuesta(++), caracterizado por un apoyo muy notable de los entes territoriales (gobierno nacional, departamental y alcaldías), quienes se muestran muy empeñados en jalonar el sector piscícola definiendo una visión para el sector e involucrando acciones desde sus planes de desarrollo fundamentados en un plan de acción al 2020 concertado entre los distintos actores, traduciéndose en inversiones en tecnológica e infraestructura y crecimiento de las organizaciones y de cada uno de los eslabones que constituyen la cadena piscícola (empresas productoras de alevinos, organizaciones de productores piscícolas continentales y marítima, plantas de transformación, centros de acopio y comerciantes). Todo este apoyo unido a la academia (Universidades públicas y privadas) y al SENA permite que los productores y distintos eslabones del sector cualifiquen sus conocimientos y compartan una visión empresarial de la actividad, la cual se expresa en un alto nivel organizativo y de agremiación de los actores de la cadena piscícola.

En este escenario, se ha visionado la conformación de la cadena a partir de dos niveles, el primer nivel (nivel 1) está representado gremios focalizados y operando en mini cadenas que se han conformado atendiendo a las particularidades biogeográficas de las cinco (5) subregiones del departamento en que se encuentra, y se ha avanzado en la investigaciones y producciones piscícolas marítimas en el Golfo de Morrosquillo. El segundo nivel (nivel 2), está representando por delegados de las diferentes minicadenas subregionales quienes conforman la cadena productiva piscícola departamental conjuntamente con representantes de los entes territoriales de orden departamental y nacional y universidades, y tiene esta agremiación la función de coordinar, dinamizar y gestionar el crecimiento y desarrollo tecnológico y competitivo de la «Cadena Productiva Piscícola para el Departamento de Sucre» a nivel nacional e internacional. Generando, una dinámica de beneficios mutuo y aplicando una política solidaria y de comercio justo entre todos los actores, fundamentado en una apuesta donde «todos ponen y todos ganan»

\section{EI Paraíso en tinieblas - Escenario Alterno 1:}

Asociaciones de pequeños productores piscícolas de Sucre compitiendo en mercados locales y de la costa Caribe.

Considerado como el panorama medio o regular(+ -), en el que los actores del sector piscícola (pequeños productores y asociaciones piscícola continentales) del departamento de sucre están unidos con la academia (Universidades), existe un adelanto tecnológico implementado e los eslabones de la cadena pero con un deficiente apoyo de los entes territoriales (gobernación y alcaldías), lo que no es suficiente para consolidar las minicadenas focalizadas por las subregiones ni para apoyar e impulsar al sector hacia una agremiación sólida y de carácter competitivo en el mercado nacional e internacional, su alcance productivo y comercial está limitado a el mercado local en el mismo departamento y en la Costa Caribe.

En este escenario existe una agremiación departamental a funcional, que no opera y sin apoyo de los entes territoriales. Generando mínimos beneficios a los distintos eslabones de la cadena, para los productores, pero muy representativa para los acopiadores y comercializadores. La apuesta «todos ponen y todos ganan», es débil y con mínimos ingresos para las bases.

Sueños al Aire - Escenario Catastrófico: Asociaciones de productores piscícolas produciendo sus peces de manera tradicional y comercializándolos en sus corregimientos y municipios, con poca tecnificación y sin agremiaciones. 
Considerado como el panorama más negativo o escenario catastrófico(- -), En este escenario la situación político-administrativa se vuelve de espalda al sector piscícola, no tiene en cuenta las partidas presupuestal ni incluye dentro de sus planes y proyectos acciones encaminadas a fortalecer y proyectar al sector piscícola. Lo que conlleva que los pequeños productores continúen haciendo las prácticas de producción artesanal que hasta hoy aun realizan sin la aplicación de tecnologías, actuando de manera individual y sin una visión empresarial del negocio, lo que debilita cada año más el sector.

De igual forma la academia y el SENA no muestra interés en apoyar al sector ni lo involucra dentro de sus acciones, lo que incrementa la brecha tecnológica y desarrollo organizativo entre los productores. Los piscicultores continúan dispersos y no muestran voluntad alguna por articularse y asociarse, no existe en ellos la búsqueda de un beneficio para el sector piscícola y la cobertura de sus mínimos productos se limita a cubrir los mercados en sus corregimientos y municipios, sin que exista altos volúmenes excelente calidad de sus productos. En este escenario es poco o casi nulo el valor agregado al producto, ya que se carece de infraestructura y tecnologías en las comunidades y los mayores ingresos se los llevan los intermediarios del departamento y de departamentos vecinos. No existe una dinámica de beneficio para ninguno de los actores, ni tampoco solidaridad, ni cooperación de los actores institucionales, generando un cambio en la apuesta, aquí «nadie pone y nadie gana»

\section{El Paraíso Oscuro - Escenario Alterno 2:} Apoyo político administrativo de los gobiernos departamentales, locales y de la academia, y falta de organización y articulación de los pescadores y otros actores.

Considerado como el panorama medio o regular (+ -), donde existe toda un acompañamiento desde las variables político administrativas, ex- presado en el empeño y voluntad de los entes territoriales (gobernación y alcaldías), quienes han definido un plan de acción para la puesta en marcha de la cadena productiva piscícola e involucra en sus planes de desarrollo partidas presupuestales para la gestión y fomento de la piscicultura. Genera los espacios para que los distintos actores se encuentren y se puedan consolidar alianzas y definan planes de acciones orientados a la consolidación de las mini cadenas focalizadas por las subregiones y para la consolidación de una agremiación sólida y de carácter competitivo en el mercado nacional e internacional. Sin embargo, los actores del sector piscícola (pequeños productores y asociaciones piscícola continentales) del departamento de sucre no muestran interés en asociarse ni en fortalecer sus cualidades como piscicultores.

En este escenario no existen agremiaciones en las minicadenas subregionales ni tampoco a nivel departamental. La producción y comercio de peces tiene adelantos tecnológicos pero no hay articulación de los proceso ni de las organizaciones, lo que no permite generar valor agregado a los distintos eslabones de la cadena, ni para los productores. Además, no existe ningún interés de los piscicultores por construir y poner en marcha la cadena productiva piscícola del departamento, a pesar de todos los esfuerzos y garantías de los gobiernos locales, nacionales, de la academia y del SENA. La apuesta «todos ponen y todos ganan», es muy débil y con mínimos ingresos para las bases.

\section{Conclusiones}

Teniendo en cuenta el respectivo análisis de los escenarios podemos concluir, que la apuesta planificadora es por el escenario: «el paraíso soñado», porque además de combinas las dos grandes variables la Sociocultural - Tecnológica y Político - Administrativa posibilita la oportunidad para que el sector piscícola del departamento de Sucre se tecnifique, 
crezca y se consolide como un sector competitivo en los mercados regionales, nacionales e internacionales.

De igual forma se plantea que este proceso debe inicialmente fortalecerse en el desarrollo tecnológico y la investigación sobre el sector y debe focalizarse por mini cadenas subregionales, para luego pasar a un nivel organizativo gremial departamental, como estrategia para que este sector se consolide como un verdadero modelo de desarrollo tecnológico y socioeconómico para el departamento y la región Caribe. El método prospectivo nos enseña que la construcción de escenario es una metodología sistémica en el que los resultados de una actividad se convierten en el insumo para consecución del próximo paso. En este sentido, la formulación de preguntas claves en la encuesta, se convierte en la parte fundamental para identificación de variables claves, actores y en la construcción del escenario apuesta para el futuro del sector piscícola en el Departamento de Sucre.

El escenario apuesta es «El Paraíso Soñado», ya que permite que los dos grupos de variables sociocultural - tecnológica y la político - administrativa se integraran e interactuaran complementándose y propiciando alianzas y articulación entre las mismas, hacia la construcción de la cadena. La construcción del plan prospectivo para el sector piscícola al 2020 permite concluir que es posible la puesta en marcha de la cadena piscícola del departamento de Sucre, si iniciamos con la realización de diagnósticos reales sobre el sector y su estado, la conformación de mini cadenas subregionales y si existe un compromiso y visión conjunta de los diferentes actores y de las instituciones nacionales, en el que se visione este sector como un pilar de desarrollo socioeconómico para el departamento de cara al desarrollo tecnológico y a la globalización del futuro. Diseñar un plan de contingencia para el sector piscícola del Departamento de Sucre, debe identificar y definir las acciones de tipo administrativas y técnicas que se han de implementar por las administraciones locales y departamentales, como también por las asociaciones y agremiaciones del sector, ante una situación inusual que puede alterar su funcionamiento normal; en este caso, el evento a prever son los efectos desastrosos y grandes pérdidas originadas por la presencia de los fenómeno climatológicos de la niña y por el fenómeno del niño.

\section{Referencias}

Chavarro, Luz. Martínez Carmen. (2002). Modulo Prospectiva. Bogotá: Unad.

Colciencias. (2006). Agenda Prospectiva de Ciencia, Tecnología e Innovación del Departamento de Sucre. Sincelejo Sucre, Visión 2005 - 2020, Colciencias, Unisucre, CECAR, SENA; Cámara de Comercio, septiembre 5 de 2006 .

Franco, Carlos. (2003). Iniciación a la prospectiva Estratégica. Bogotá: UNAD.

Godet Michel. (2000). Caja de herramientas de la Prospectiva Estratégica. Cuaderno \# 5. Cuarta Edición actualizada abril de 2000.

Mera Rodríguez, Carlos William. (2005). Modulo Prospectiva Estratégica. Bogotá: UNAD.

Ministerio de Agricultura y Desarrollo Rural y el Observatorio de Ciencia y Tecnología. (2008). Informe General del Estudio de Prospectiva tecnológica de la cadena Colombiana de la Tilapia en Colombia.

Ministerio de Agricultura y Desarrollo Rural. (2005). Acuerdo de Competitividad de la Cadena de la Piscicultura en Colombia, y Acuerdo Regional de Competitividad de la Cadena Piscícola en el Departamento del Meta, octubre de 2005.

Mojíca, Francisco. (2011). El modelo prospectivo llevado a la práctica. Primera versión. Disponible en: http:// es.scribd.com/doc/75128269/EL-MODELO-PROSPECTIVO-LLEVADO-A-LA-PRACTICA-ONCYT-CAB-PorFrancisco-J-Mojica\#scribd

Mojíca, Francisco. (1992). Prospectiva, Técnicas para visualizar el Futuro,Bogotá: Legis.Universidad de Sucre. (2005). Diagnóstico Piscícola del departamento de Sucre, en el Marco del inventario Acuícola Nacional, convenio Instituto Colombiano Agropecuario ICA - Universidad de Sucre 2005 . 2006 